\title{
Mögliche Auswirkungen von Pharmacogenomics auf Heilungskosten und Versicherungen
}

\author{
W. Oggier
}

Im ersten Abschnitt werden einige ökonomische Begriffe und Grundlagen zum schweizerischen Gesundheitssystem erörtert, welche im Zusammenhang mit Pharmacogenomics für Leistungserbringer, Versicherungen, Pharma- und Biotechnologiesektor von Bedeutung sein können.

In den anschliessenden Abschnitten werden Resultate aus den durchgeführten Interviews zu den Auswirkungen auf die direkten (Heilungs-)Kosten im Gesundheitswesen sowie die Auswirkungen auf die Reduktion der Kosten durch unerwünschte Nebenwirkungen dargestellt.

Die Ergebnisse dieser Expertenbefragung dienen als Basis für die Erarbeitung weitergehender Überlegungen in Form von Empfehlungen. Dazu wurde auch weitere ausländische Literatur ausgewertet. Im Rahmen dieser Ausführungen werden aus gesundheitsökonomischer Sicht einige Empfehlungen zur Wissenschafts- und Bildungs-, zur Wirtschafts- sowie zur Gesundheits- und Sozialpolitik gemacht.

1 Greiner W. Die Berechnung von Kosten und Nutzen im Gesundheitswesen. In: Schöffski O, Glaser P, von der Schulenburg J-M (Hrsg.). Gesundheitsökonomische Evaluationen. Berlin, Heidelberg, New York: Springer-Verlag; 1998. S. 55-67, insbesondere S. 57ff.

2 Münchener Rück (Hrsg.). Gentechnologie - eine Herausforderung für die Versicherungswirtschaft. München: Münchener Rück; 2002. S. 134.

Korrespondenz:

Dr. oec. HSG Willy Oggier

Konradstrasse 61

CH-8005 Zürich

\section{Kostenbegriff und Messinstrumente}

Wenn von Kosten im Gesundheitswesen die Rede ist, kommt der Definition des Kostenbegriffs entscheidende Bedeutung zu. Die Gesundheitsökonomie unterscheidet in der Regel drei verschiedene Dimensionen, wenn es um die Kosten geht: die direkten, die indirekten und die intangiblen Kosten. $\mathrm{Zu}$ den direkten Kosten und Nutzen wird der Ressourcenverzehr gezählt, der unmittelbar mit der Anwendung bzw. Ausführung der ärztlichen, pflegerischen, therapeutischen Behandlung verbunden ist (Kosten) bzw. vermieden werden kann (Nutzen). Zur Berechnung der indirekten Kosten wird in der Regel nach dem sogenannten Humankapitalansatz vorgegangen. Die Bezeichnung des Ansatzes unterstellt, dass Gesundheitsausgaben aus volkswirtschaftlicher Sicht immer auch Investitionen in die Erhaltung der Berufsausübungsfähigkeit der Patienten, also in das Humankapital darstellen. Die indirekten Kosten einer Krankheit sind also so gross wie der Verlust an Arbeitspotential, der einer Volkswirtschaft durch krankheitsbedingte Absenzen oder eingeschränkte Leistungen am Arbeitsplatz entsteht [1]. Zur dritten Dimension, den intangiblen Kosten, vermögen die Gesundheitsökonomen in der Regel keine Aussagen zu machen. Die intangiblen Kosten umfassen nämlich vor allem Gefühle wie Angst, Schmerzen, Trauer der kranken Menschen und ihrer Freunde und Bekannten. Diese Kosten las- sen sich mit den heute gängigen Verfahren nach wie vor nicht in Franken und Rappen bewerten, auch wenn neuere Forschungsarbeiten vermehrt in dieser Richtung tätig werden.

Für die folgenden Überlegungen geht es in erster Linie um die direkten Kosten und deren Veränderungen durch Pharmacogenomics. In diesem Zusammenhang lassen sich zwei unterschiedliche Thesen unterscheiden, welche folgendermassen dargestellt werden können [2]:

- Die Kompressionsthese geht davon aus, dass Menschen bei steigender Lebenserwartung ihr Leben insgesamt auch in «besserer» Gesundheit verbringen. Ein Grossteil des Lebenszyklus wird frei von schweren oder chronischen Erkrankungen verbracht (Compressed-Morbidity-Modell).

- Die Medikalisierungsthese besagt, dass zusätzliche Lebensjahre eher mit Krankheit oder mindestens mit chronischen Beschwerden einhergehen und nicht in völliger Gesundheit verbracht werden. Nach diesem Modell wäre bei steigender Lebenserwartung mit einem starken Schub auf der Ausgabenseite zu rechnen. Ein Beispiel hierfür können Behandlungsfortschritte bei chronischen Krankheiten wie Herzkrankheiten oder Krebs sein, die zwar die Sterblichkeit verzögern, aber langjährige, kostenintensive Therapie- und Rehabilitationsmassnahmen notwendig machen.

Jüngere Arbeiten scheinen für beide Thesen empirische Belege finden zu können. Die Auswirkungen können dabei auch von Leistungsbereich $\mathrm{zu}$ Leistungsbereich variieren. Aus versicherungsökonomischer Sicht kommt der Entwicklung von Schlüsseltechnologien wie etwa der Gentechnologie hin zur Kompression bzw. zur Medikalisierung daher besondere Bedeutung zu.

\section{Grundzüge des schweizerischen Krankenversicherungssystems}

Das schweizerische Gesundheitswesen unterscheidet für den Krankheitsfall zwischen einer Grundversicherung, welche ihre gesetzlichen Grundlagen im KVG hat, und privaten Zusatz- 
versicherungen, welche dem Gesetz über den Versicherungsvertrag (VVG) unterstellt sind. Im Gegensatz zu anderen Ländern sind Zusatzversicherungen in der Schweiz komplementärer und nicht ersetzender Natur. Personen, welche eine Zusatzversicherung abschliessen, können sich demnach nicht der Bezahlung der Grundversicherungsprämie entziehen. Anders als beispielsweise in Deutschland kann man sich also nicht $\mathrm{ab}$ einer bestimmten Einkommensgrenze von der gesetzlichen Krankenversicherung und damit von der Solidargemeinschaft verabschieden, indem man zu einer privaten Krankenversicherung wechselt. Auch Beamte haben - mit Ausnahme gewisser gerade aktiv Dienst leistender Militärs - eine Grundversicherungsprämie zu entrichten.

Grund- und Zusatzversicherungen sind mit der Einführung des KVG am 1. Januar 1996 vom Gesetzgeber juristisch getrennt worden. Einige wesentliche Unterschiede lassen sich auf folgende Art und Weise darstellen [3]:

( 9 September 1996, aktualisiert.

Tabelle 1

Wichtige Unterschiede zwischen Grund- und Zusatzversicherungen.

\section{Grundversicherung nach KVG \\ Grundlage sind Gesetz und Verordnungen \\ Obligatorium und Aufnahmepflicht durch die Versicherer}

Es besteht ein fest definierter gesetzlicher Leistungskatalog mit Einschluss der Mutterschaft

Finanzierung nach Umlageverfahren (die Ausgaben werden laufend durch Einnahmen Prämien und Kostenbeteiligung - gedeckt)

Einheitsprämien pro Versicherer und Region. Prämienreduktion für Kinder und Jugendliche. Prämienverbilligung

Die Versicherten können den Versicherer mit einer Kündigungsfrist von einem Monat auf Semesterende ohne Nachteile wechseln. Der Versicherer kann dem Versicherten nicht kündigen. Ein Ausschluss ist kaum möglich. Leistungssistierung bei ausstehenden Prämien, bis die Schulden bezahlt sind

Keine Vorbehalte bei Versicherungswechsel

Unbeschränkte Versicherungsdauer Prinzip der Gegenseitigkeit und Gleichbehandlung

Verjährung von Leistungsansprüchen nach

fünf Jahren

Keine Kollektivversicherungen mehr

\section{Zusatzversicherung nach VVG}

Grundlage ist der Vertrag mit den allgemeinen Versicherungsbedingungen (AVB)

Keine Aufnahmepflicht; Ausnahme: Bisherige Zusatzversicherte nach altem Recht haben beim gleichen Versicherer Anrecht auf einen Vertrag, der die bisherigen Leistungen abdeckt

Der Versicherer bestimmt, welche ergänzenden Leistungen zur Grundversicherung er anbieten will. Mutterschaft auf Antrag

Keine Vorschriften. In der Praxis: Bedarfsdeckungs- oder Kapitaldeckungsverfahren oder eine Mischform der beiden (Rückstellung für spätere Verpflichtungen oder Ansparen von Kapital für Risiken im Alter)

Keine Vorschriften für Prämiengestaltung. In der Praxis fördert der Wettbewerb aber risikogerechte Prämien

Beide Parteien können in einem Versicherungsfall sofort (bis vierzehn Tage nach Zahlung) oder auf Vertragsende mit einer dreimonatigen Kündigungsfrist kündigen (ohne Kündigung stillschweigende Vertragsverlängerung um ein Jahr). Viele Versicherer verzichten einseitig auf ihr Recht, im Versicherungsfall zu kündigen

Unbeschränkte Vorbehalte oder Leistungsausschlüsse möglich

Die Dauer der Verträge kann limitiert werden Prinzip von Treu und Glauben

Verjährung von Leistungsansprüchen nach zwei Jahren

Kollektivversicherungen sind möglich
Wegen dieser Unterschiede kann davon ausgegangen werden, dass sich für Versicherer in der Grund- bzw. in den Zusatzversicherungen unterschiedliche Fragen stellen. Im Bereich der Zusatzversicherungen dürfte insbesondere die Frage vermehrt gestellt werden, ob eine entscheidende Grundlage der Versicherung, nämlich die Unkenntnis der Versicherten über ihren aktuellen und zukünftigen gesundheitlichen Status, gewährleistet bleibt und ob es zu einer gezielten Verschiebung nicht versicherbarer Personen oder schlechter Risiken unter Umgehung der Offenbarungspflicht kommt. Befürchtet wird, dass Versicherte versuchen, ihr individuelles Risiko abzuschätzen, und sich im Fall hoher Risiken finanzielle Vorteile verschaffen [4]. Denkbar wäre aber auch die umgekehrte Situation, indem sich Versicherte testen lassen und mit ihren positiven Resultaten beim Versicherer durch Offenlegung ihrer guten Risiken eine niedrigere Prämie realisieren wollen.

Im Bereich der Grundversicherung dürfte es vermehrt um die Frage gehen, wie der medizinische Fortschritt noch finanziert werden soll und wie die vorhandenen Ressourcen am effizientesten eingesetzt werden. Zur Beantwortung dieser Fragestellung von Bedeutung ist auch die unten näher analysierte Frage, welche Auswirkungen Pharmacogenomics auf die Heilungskosten bzw. die gesamten Gesundheitskosten zeitigt. Für das schweizerische System stellt sich zudem die Frage, welche Anteile allfälliger Einsparungen der Grundversicherung verbleiben und welche Anteile anderen Bereichen zufliessen. Im Gegensatz etwa zur heutigen gesetzlichen Krankenversicherung in Deutschland übernimmt der Grundversicherer in der Schweiz den Erwerbsausfall aus Krankheit kaum oder in der Regel gar nicht. Denn Taggeldversicherungen sind in der Regel nach VVG ausgestaltet und werden oft im Rahmen von Tarifverhandlungen zwischen den Sozialpartnern vereinbart. Reduktionen der Arbeitsunfähigkeitszeiten durch medizintechnische oder pharmakogenetische Fortschritte werden im schweizerischen Gesundheitssystem somit bei Leistungspflicht durch die Grundversicherung vom Krankenversicherer bezahlt. Profiteur dieser Fortschritte ist aber oft nicht der Finanzierer Krankenversicherer, sondern der Arbeitgeber, welcher die Wertschöpfungsgewinne realisieren kann. 


\section{Auswirkungen auf die direkten (Heilungs-)Kosten im Gesundheitswesen}

Um wenigstens eine erste qualitative Annäherung an die Auswirkungen auf die direkten (Heilungs-)Kosten zu realisieren, wurden sechs halbstrukturierte mündliche und schriftliche Interviews mit unterschiedlichen nationalen und internationalen Akteuren auf der Pharmaund auf der Versichererseite durchgeführt.

Kurzfristig ergeben sich zwischen den befragten Interviewpartnern aus der Pharma- bzw. der Versicherungsbranche keine Differenzen. Sie gehen davon aus, dass sich kurzfristig kaum Veränderungen bzgl. der Gesundheitskosten ergeben werden. Dieses einstimmige Urteil wurde u.a. auf folgende Arten begründet:

- Wegen der geringen Zahl ist nicht mit nennenswerten Veränderungen zu rechnen.

- «Optimistisch gedacht, kommt es zu keiner Veränderung.»

- Die Technologien sind nicht so teuer, denn das DNA-Chipping ist automatisiert. Entscheidend bei Kostendiskussionen sind die Monatstherapiekosten für die Genmedikamente.

- «In dieser kurzen Zeitspanne wird es noch keine Auswirkungen geben.»

Die Antworten für den langfristigen Bereich lassen dagegen vermehrt den Gegensatz zwischen der Kompressions- und der Medikalisierungsthese erkennen. Bei dieser Fragestellung kommen die Gräben zwischen den Vertretern der Pharma- und der Versicherungsindustrie unter allen im Fragebogen gestellten wirtschaftlichen Fragen am deutlichsten zum Ausdruck. Alle Vertreter der Pharmaindustrie sprachen sich für den Verringerungseffekt aus, die Mehrheit der Versicherungsvertreter äusserte sich klar und kategorisch in Richtung einer Verteuerung.

Für eine Verringerung wurden folgende Argumente angeführt:

- «Die Medikamentenbehandlungen werden effektiver und effizienter. Es reduzieren sich die aufwendigen Nebenwirkungen.»

- Gezieltere Therapien werden möglich.

- Für unnötige Therapien wird weniger Geld ausgegeben.

- Unnötige Hospitalisationen können vermieden werden.

- Die Standardisierung der Behandlungen senkt die Kosten, kurzfristig ist die Technologie noch teurer.

Für eine Verteuerung wurden folgende Argumente angeführt:
- Es kommt zu einer leichten Verteuerung. Zwar kann durchaus auch mit Substitutionsund Einsparungseffekten (Vermeidung von Medikationen, Hospitalisationen) gerechnet werden. Es ist aber wohl vor allem mit zusätzlichen Anwendungen (zusätzliche Diagnostik und neue Therapiemöglichkeiten) zu rechnen. Es ist kaum davon auszugehen, dass sich zusätzlicher Nutzen in geringeren Kosten widerspiegeln wird.

- «Mittelfristig werden die Kosten verringert durch gezieltere Therapien und Prävention von teuren Krankheiten. Zudem wird das Therapiescreening bei teuren Krankheiten verhindert. Die Medikamente werden aber sehr viel teurer sein und diese Einsparung wettmachen.»

- «Langfristig wird die Lebensdauer der Menschen verlängert, was die Kosten verteuert. $\mathrm{Zu}$ erwarten ist sogar, dass die Pflegebedürftigkeit zunehmen wird (mit entsprechenden Kostenfolgen), da die degenerativen Erkrankungen sich nicht alle aufhalten lassen.»

\section{Auswirkungen auf die Reduktion der Kosten durch unerwünschte Neben- wirkungen}

Einer der Hauptnutzen der Pharmacogenomics wird von deren Promotoren in der Reduktion unerwünschter Nebenwirkungen gesehen. Aus diesem Grund wurde auch eine Frage gestellt, um zu eruieren, wieweit diese Nutzeneffekte allgemein anerkannt sind.

Die Interviewpartner sehen kurzfristig eine kleine oder sehr kleine Auswirkung. Damit decken sich die Antworten auf diese Frage mit jenen zu den kurzfristigen Resultaten bei den Auswirkungen auf die direkten (Heilungs-) Kosten.

Für einen kleinen bzw. sehr kleinen Einfluss wurden u.a. folgende Argumente angeführt:

- Kurzfristig ist von einer geringen Ausbreitung auszugehen.

- «Es ist zu früh für allfällige Wirkungen.»

Die Antworten bzgl. der langfristigen Auswirkungen verhalten sich diametral entgegengesetzt $\mathrm{zu}$ jenen der kurzen Frist. Die Interviewpartner erwarten grosse oder sehr grosse Auswirkungen. Dafür wurden u.a. folgende Argumente angeführt:

- Der Begriff «sehr gross» ist noch zu klein. Es besteht das Ziel, 10 Prozent der 26 Prozent Nebenwirkungen gravierender Art mit der ersten Generation an Tests zu beseitigen. 
- Die Tests sind am Anfang teuer, nachher nivellieren sich die Preise. Das Zeitfenster könnte allerdings 15 bis 20 Jahre betragen. Ähnliche Zeitspannen hat man in der Immunchemie und bei Fertilitätstests feststellen können.

- «Wenn man diesen Effekt nicht hinkriegt, ist die ganze Technologie fragwürdig.»

- Das ökonomische Ausmass von unerwünschten Arzneimittelnebenwirkungen wurde bislang unterschätzt. In Deutschland wird die Zahl medikamentenbedingter Todesfälle auf 16000 , die ernster Nebenwirkungen auf jährlich über 120000 Fälle (Deutsches Ärzteblatt, 13. Juni 2003) geschätzt. Da die Pharmaindustrie sich schon aus wirtschaftlichen Gründen auf pharmakogenetisch unterstützte Behandlungen häufiger Erkrankungen konzentrieren wird, dürfte mit einem hohen Einsparpotential zu rechnen sein. Mittelfristig ist nicht auszuschliessen, dass ganze pharmagenetische Profile/Steckbriefe der jeweiligen Patientinnen und Patienten die behandelnden Ärztinnen und Ärzte bei der Auswahl der Medikation verschiedenster Erkrankungen unterstützen werden.

- Mit der breiteren Anwendung gewinnen auch die Auswirkungen an Gewicht.

- Durch die individualisierte Therapie sollten die Nebenwirkungen reduziert und damit Kosten eingedämmt werden. Zudem werden nur Patientengruppen, die Responser sind, therapiert. Nebenwirkungen wegen unwirksamer Therapien entfallen. Vielleicht entstehen aber auch Interaktionen, die mit «normalen» Medikamenten noch nicht voraussehbar sind.

Die Begründungen zeigen auf, dass zwar Einigkeit im Bedeutungsgrad der Auswirkungen besteht. Welcher Art diese Auswirkungen aber sein werden, bleibt zwischen den Interviewpartnern kontrovers. Die einen argumentieren zuweilen fast credohaft nach dem Motto «Es muss einfach funktionieren, sonst hätte das Ganze ja keinen Sinn. Und was nicht Sinn macht, darf einfach nicht sein». Die anderen vermuten teilweise neue Interaktionen mit womöglich neuen Nebenwirkungen und damit verbunden einen Schneeballeffekt, nach dem Motto «Solange nichts bekannt ist, muss ich vom Schlimmsten ausgehen».

\section{Ökonomische Auswirkungen auf die Versicherungen}

Kurzfristig wird von allen Interviewpartnern davon ausgegangen, dass die Auswirkungen als klein oder sehr klein zu bezeichnen sind. Typische Aussagen für diese Auffassungen waren etwa:

- Die Anzahl der neu zu finanzierenden Medikamente, die Pharmakogenetik anwenden, ist hinsichtlich Quantität noch sehr klein: Gleevic (chron. myel. Leukämie), Tacrin (Alzheimer), Herceptin (Brustkrebs), Interferon (Hepatitis C).

- Aus Finanzierungsoptik dürfte sich in den nächsten drei Jahren nicht viel verändern. Pharmacogenomics befindet sich noch in den Kinderschuhen und ist vermutlich versicherungsmässig noch nicht unmittelbar relevant.

Die Antworten bezüglich der langfristigen Auswirkungen zeigen ein anderes Bild. Mehr als 80 Prozent der befragten Personen erwarten in dieser Zeitspanne einen sehr grossen bzw. grossen Einfluss. Als Begründungen wurden u.a. angeführt:

- Die mit Pharmacogenomics aufgeworfenen Fragen werden für die Sozialversicherungen und die Krankenversicherung im speziellen von vitaler Bedeutung sein. Es geht u. a. um das Gefühl des Ausgegrenztseins von Versicherten.

- «In der obligatorischen Grundversicherung werden langfristig Kosten durch diese Entwicklung entstehen, die das bisherige Versicherungswesen auf den Kopf stellen. Die Kosten, die gespart werden durch effizientere Therapien, werden durch weitere Mengenausweitung und sehr teure Medikamente weit übertroffen. Neue und teure Medikamente werden schneller auf dem Markt sein und vermehrt präventive Behandlungen leistungspflichtig, deren Kosten/Nutzen-Effekt erst nach vielen Jahren beobachtet werden kann.»

- «Die Zusatzversicherungen stehen unter dem Anspruch, ökonomisch zu arbeiten. Dies zwingt sie, die Prämien anhand des Risikopotentials des Kunden zu berechnen. Der Druck, neue Erkenntnisse über das Gefahrenpotential des einzelnen zu erfahren, wird stärker sein als der Schutz des einzelnen. Gute Risiken werden einfach und günstig Zusatzversicherungen abschliessen können oder im Extremfall Rabatte verlangen. Schlechte Risiken werden sich kaum mehr 
privat versichern können, ausser zu einem hohen Preis.»

- In diesem Zeitraum muss beispielsweise damit gerechnet werden, dass in der antidepressiven Therapie pharmakogenetische Tests $\mathrm{zu}$ selektiven Serotoninrezeptorhemmern entwickelt werden. Bei einer Bevölkerungsprävalenz zur Depression von 10 Prozent dürfte sich dies auch für die Kostenträger auswirken: bessere Compliance, weniger Nebenwirkungen, mehr Therapieerfolge (20 bis 50 Prozent der SSRI schlagen bei Behandlungen nicht an!), weniger Nachbehandlungen und Komplikationen. Weitere Beispiele sind die Behandlung HIV-Infizierter durch individualisierte genotypabhängige Therapieregime oder die individualisierte, vom jeweiligen Expressionsprofil des Tumors gesteuerte Chemotherapie.

- Es wird die Frage gestellt werden, wie es mit der Kostenerstattung durch Krankenversicherungen bei Medikamenten steht, die nachweislich beim Versicherten nicht wirken können.

Die Aussagen machen deutlich, dass zwar mehr oder weniger Einigkeit besteht über das grundsätzliche Ausmass der Auswirkungen. Unterschiede bestehen aber in der Begründung dieses Ausmasses. So besteht beispielsweise keine Einigkeit darüber, ob Grund- und Zusatzversicherung gleich oder unterschiedlich von diesen Entwicklungen betroffen sind.

\section{Empfehlungen}

Die ökonomischen Grundfragen im Bereich Pharmakogenetik und Pharmakogenomik unterscheiden sich nicht von den allgemeinen ökonomischen Fragestellungen, die sich im Bereich anderer genetischer Untersuchungen, der Entwicklung neuer Medikamente oder medizintechnischer Verfahren stellen. Aus diesem Grund darf es auch nicht überraschen, dass ökonomische Überlegungen bei Pharmacogenomics methodisch nach der gleichen Vorgehensweise erörtert werden wie beispielsweise bei der Medizintechnologiebewertung. Pharmacogenomics bietet daher auch in der Regel nicht besonders komplexe oder schwierig zu beantwortende grundsätzliche Fragestellungen. Sie könnte aber bestimmte Grundsatzfragen wegen ihrer von den interviewten Personen erwarteten zunehmenden ökonomischen Bedeutung in der $\mathrm{Zu}$ kunft vermehrt und in intensiviertem Ausmass stellen.
Dazu gehört beispielsweise die Frage, wie sichergestellt werden kann, dass die erhofften positiven Folgen pharmakogenetischer Entwicklungen möglichst vollständig und effizient für die schweizerische Volkswirtschaft und deren Bewohnerinnen und Bewohner zur Geltung kommen können. Aus ökonomischer Sicht verdient in diesem Zusammenhang insbesondere die Verzerrung der finanziellen Anreizmechanismen im Rahmen des schweizerischen Sozialversicherungssystems besondere Beachtung und sollte inskünftig weitgehend vermieden werden.

Im Bereich der Sozialversicherungen und der obligatorischen Krankenpflegeversicherung im speziellen stellt sich bei der Neuzulassung von Pharmaka unter anderem die Frage, wie geeignete Indikationsregeln entwickelt werden können, um zu verhindern, dass pharmakogenomisch hergestellte Medikamente unspezifisch eingesetzt werden bzw. wegen des geringen Einsatzes durch das entsprechende Personal vermeidbare qualitative Mängel entstehen.

Pharmacogenomics dürfte aber auch die schweizerische Wissenschafts- und Bildungspolitik vermehrt vor grundsätzliche Fragen stellen. Genauso wie in der Gesundheitspolitik fehlt auch in diesem Bereich über weite Strecken eine Definition klarer Ziele, die mit entsprechenden staatlichen bzw. parastaatlichen Tätigkeiten erreicht werden sollen. Das ökonomisch grösste Defizit besteht in diesem Bereich bei der Festlegung quantitativer Zielgrössen.

Vor dem Hintergrund der gemachten Ausführungen lassen sich für die versicherungsspezifischen Aspekte u.a. folgende Empfehlungen* ableiten:

- Die engen Spielräume für eine wirksame Steuerung des Leistungsgeschehens können am ehesten noch im Stadium der Einführung einer neuen Technik ausgefüllt werden. Frühere Erfahrungen mit anderen Verfahren legen den Schluss nahe, dass eine Technik, die ihre Diffusion bereits vollzogen hat, selbst dann nur schwer aus dem Leistungskatalog zu entfernen ist, wenn sich ihre Effektivität und Effizienz als problematisch erwiesen hat. Durch den Aufbau eines verschärften Selektionsdrucks für die allgemeine Abdeckung durch solidarisch finanzierte Systeme soll die breite Einführung ineffizienter Techniken vermehrt vermieden werden. Im Gegenzug wird damit angesichts des sich abzeichnenden Finanzierungsdrucks Innovationen, die eine nachweisbar positive Kosten-Nutzen- und Nutzen-Risiko-Bilanz aufweisen, eine bessere Chance gegeben. 
- Die heute verwendeten gesundheitsökonomischen Instrumente bei Antragsverfahren zur Aufnahme in den Leistungskatalog der obligatorischen Grundversicherung nach KVG greifen zu kurz. Neben medizinischen und ökonomischen sollten zwingend auch ethische und soziale Auswirkungen der Technik zu kalkulieren versucht werden. Ziel dieser Anpassungen muss sein, eine kontrollierte Prüfung, Einführung und Diffusion sicherzustellen, welche zweierlei verhindert: die fast automatische Anwendung, Ausbreitung und Wachstumsdynamik einer ineffektiven Technik sowie das Versagen gesundheitspolitischer Interventionen bei der Streichung ineffektiver medizinischer Techniken aus dem Leistungskatalog, die einmal darin Eingang gefunden haben.

- Die eigentlichen pharmakogenetischen Kosten dürften mittelfristig weniger durch die Diagnostik selbst entstehen, sondern durch die Nachfragekaskaden, die ein positiver Test in angrenzenden medizinischen Leistungsangeboten (weitere Diagnostik, engmaschige Früherkennung, Prävention, prophylaktische Intervention, Therapiekontrolle) auslöst. Aus der Perspektive der obligatorischen Krankenversicherung dürfte es daher wichtig sein, für die medizinischen Anschlusshandlungen an einen prädiktiven Gentest geeignete Indikationskriterien zu entwickeln und durchzusetzen.

Hinzu kommen kann, dass neue Medikamente mit teilweise begrenztem oder im Verhältnis zur Preisdifferenz äusserst geringfügigem Mehrnutzen bewährte ältere Medikamente aus dem Markt drängen, weil es ökonomisch lukrativer sein kann, die alten Medikamente aus dem Markt zu nehmen.

Darüber hinaus sollten für die schweizerische Situation u.a. folgende Aspekte näher analysiert werden:

- Mit der Einführung des KVG sind die Grundund Zusatzversicherung im Bereich der Krankenversicherung ganz bewusst getrennt und nach unterschiedlichen Prinzipien gestaltet worden. Solange das KVG eine gut ausge- baute Grundversorgung sicherstellt und damit Phänomene der adversen Selektion zu reduzieren vermag, ergibt sich im Bereich der Zusatzversicherungen kaum Handlungsbedarf. Denn hier sollen die Wettbewerbsprinzipien zum Tragen kommen. Aus dieser Optik kommt im gegenwärtigen Zeitpunkt einzig dem Problem des Informationsvorsprungs von Versicherten gegenüber Versicherern besondere Bedeutung zu.

- Um die erhofften positiven Folgen pharmakogenetischer Entwicklungen möglichst vollständig und effizient für die schweizerische Volkswirtschaft zur Geltung zu bringen, sollten Verzerrungen im Finanzierungssystem der sozialen Sicherung weitgehend vermieden werden. Solche Verzerrungen treten u.a. dadurch auf, dass Arbeitsausfallentschädigungen, Invaliditätsrenten und Todesfallkapitalien durch andere Finanzierer oder die Betroffenen selbst übernommen werden müssen. Ökonomisch gesehen sollte aber der Finanzierer bestimmter (neuer) Heilverfahren auch die Resultate des Behandlungserfolgs wie beispielsweise früherer Spitalaustritt, frühere Rückkehr an den Arbeitsplatz oder Vermeidung einer Invaliditätsrente ernten können. Einer monistischen Finanzierung (also einer Finanzierung durch einen einzigen Finanzierer) der gesamten Behandlungskette kommt deshalb besondere Bedeutung zu. Diese monistische Finanzierung darf sich aber nicht nur auf den kurativen Teil oder ausschliesslich auf die direkten (Heilungs-)Kosten beschränken. Sollen volkswirtschaftliche Bedeutung und solidarisch finanzierte Sicherungssysteme vermehrt gleichgerichtete ökonomische Signale aussenden, sind sowohl geriatrische Angebote als auch Arbeitsausfallentschädigungen durch den gleichen monistischen Finanzierer zu übernehmen. Eine solche Massnahme könnte auch beim monistischen Finanzierer zu einer verstärkten Förderung neuer medizintechnischer und pharmakogenetischer Verfahren führen, die wirksam, zweckmässig und wirtschaftlich sind. 